\title{
The Effect of Infraslow Frequency Neurofeedback on Autonomic Nervous System Function in Adults with Anxiety and Related Diseases
}

\author{
Karlien Balt ${ }^{*}$, Peet Du Toit ${ }^{1}$, Mark Smith $^{2}$, and Charl Janse van Rensburg ${ }^{3}$ \\ ${ }^{1}$ Department of Human Physiology, Faculty of Health Sciences, University of Pretoria, Pretoria, South Africa \\ ${ }^{2}$ Neurofeedback Services of New York, New York, New York, USA \\ ${ }^{3}$ South African Medical Research Council, Biostatistics Unit, Pretoria, South Africa
}

\begin{tabular}{|c|c|}
\hline Abs & act \\
\hline $\begin{array}{l}\text { Peripheral body monitoring of autonomic nervous syst } \\
\text { infraslow fluctuation (ISF) neurofeedback training. Tr } \\
\text { physiological effect on an individual that can be reve } \\
\text { biofeedback metrics that included heart rate variab } \\
\text { conductance, heart rate, respiration rate, and blood pre } \\
\text { and } 55 \text {, primarily with anxiety, were randomized into two } \\
\text { group. The experimental group completed } 10 \text { ISF neurc } \\
\text { of ANS changes was applied. The same process was } \\
\text { sensorimotor rhythm (SMR) neurofeedback training. } \\
\text { conductance ( } p<.0001) \text {, electromyography }(p=.01) \text {, } \\
=.05) \text { and blood pressure (systolic change } p=.049) \text { ir } \\
\text { seen in the control group. Conclusion. The study der } \\
\text { ANS as measured by peripheral biofeedback indicators. }\end{array}$ & $\begin{array}{l}\text { (ANS) response has been routinely applied during } \\
\text { is study hypothesized that ISF training has a distinct } \\
\text { aled by measuring autonomic function with peripheral } \\
\text { ility (HRV), muscle tension, skin temperature, skin } \\
\text { ssure. Methods. Thirty adults between the ages of } 18 \\
\text { groups: } 20 \text { in the experimental group and } 9 \text { in the control } \\
\text { feedback training sessions while continuous monitoring } \\
\text { ompleted for a control group that received one-channel } \\
\text { Results. Significant changes were seen in the skin } \\
\text { ery low frequency ( } p=.004) \text {, low frequency of HRV ( } p \\
\text { the experimental group. No significant changes were } \\
\text { nonstrated that ISF neurofeedback training impacts the }\end{array}$ \\
\hline $\begin{array}{l}\text { Keywords: neurofeedback; infraslow frequency; elec } \\
\text { electromyogram; skin conductance; breathing; heart }\end{array}$ & $\begin{array}{l}\text { cephalogram; autonomic; blood pressure; } \\
\text { ariability; inter beat interval; autonomic function }\end{array}$ \\
\hline $\begin{array}{l}\text { : Balt, K., Du Toit, P., Smith, M., \& van Rensburg, C. J. } \\
\text { system function in adults with anxiety and related disease }\end{array}$ & $\begin{array}{l}\text { ). The } € \\
\text { uroRegul }\end{array}$ \\
\hline $\begin{array}{l}\text { *Address correspondence to: Karlien Balt, MSc Physiology, } \\
\text { Student number: } 21077194 \text {, Department of Human Physiology, } \\
\text { Faculty of Health Sciences, University of Pretoria, Pretoria, South } \\
\text { Africa, 0181. Email: karlienbalt@gmail.com }\end{array}$ & $\begin{array}{l}\text { Edited by: } \\
\text { Rex L. Cannon, PhD, SPESA Research Institute, Knoxville, } \\
\text { Tennessee, USA }\end{array}$ \\
\hline $\begin{array}{l}\text { Copyright: ( } 2020 \text {. Balt et al. This is an Open Access article } \\
\text { distributed under the terms of the Creative Commons Attribution } \\
\text { License (CC-BY). }\end{array}$ & $\begin{array}{l}\text { Reviewed by: } \\
\text { Rex L. Cannon, PhD, SPESA Research Institute, Knoxville, } \\
\text { Tennessee, USA } \\
\text { Randall Lyle, PhD, Mount Mercy University, Cedar Rapids, lowa, } \\
\text { USA }\end{array}$ \\
\hline
\end{tabular}

\section{Introduction}

The body has a natural and sophisticated mechanism which ensures that there is as much physiological stability as possible while we navigate through life. The control center for this homeostatic mechanism is the autonomic nervous system (ANS) which unconsciously controls and manages heart rate (HR), breathing, blood pressure, and various other functions. It consists of the sympathetic nervous system (SNS) and parasympathetic nervous system (PNS) which complement each other and so regulate physiological processes in response to external or internal stimuli. When there is a stress reaction, the SNS will respond and shift the body from its normal state of equilibrium. Once the stimulus is over, the PNS will shift it back towards its point of equilibrium (Figure 1). If this process becomes less responsive and an individual remains in a state of increased or decreased arousal, it can lead to disease. Control of the ANS has largely been attributed to the midbrain structures and the hypothalamic pituitary adrenal axis. The contribution of behavioral networks in cortex to ANS regulation has only recently been understood (Beissner, Meissner, Bär, \& Napadow, 2013; Thayer \& Lane, 2000). 


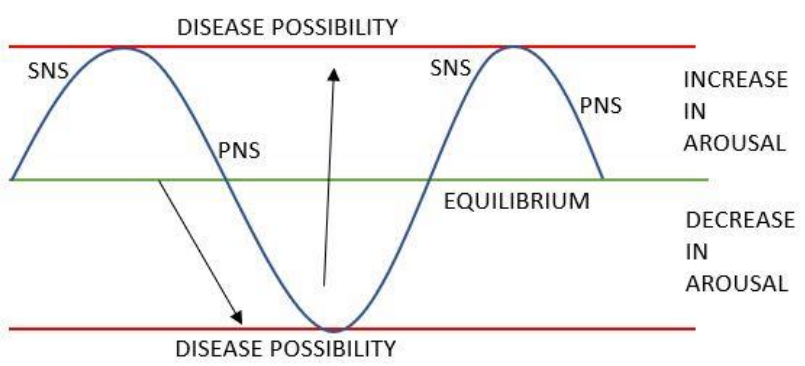

Figure 1. The autonomic nervous system and how it equilizes.

There is increasing evidence that mental and physical problems experienced by individuals are the result of flawed functional connectivity within resting state networks (Warren, Chou, \& Steklis, 2020). Importantly, the underlying functional architecture of the brain is coordinated by infraslow frequencies. This superstructure of oscillations coordinate both the connections in and decoupling between active behavioral networks (Palva \& Palva, 2012). These slow periodicities, described as less than $0.1 \mathrm{~Hz}$, were first linked with behavior by Nina Aladjalova in the 1950s in the Soviet Union. With her crude chopper stabilized amplifiers, Aladjalova associated infraslow activity with parasympathetic, reparative response (Aladjalova, 1957). Recently, the development of commercially available direct current (DC) coupled amplifiers has led to an explosion of research of human behavior and the infraslow frequencies. In the last 20 years, studies of infraslow frequencies and attention-deficit/hyperactivity disorder (ADHD), hormone response, pain, memory, sleep, and seizure, to name just a few, have been published worldwide in journals and textbooks (Alshelh et al., 2016; Broyd, Helps, \& Sonuga-Barke, 2011; Helfrich, Mander, Jagust, Knight, \& Walker, 2018; Joshi et al., 2018; Lecci et al., 2017; Marshall, Mölle, Fehm, \& Born, 2008). The centrality of the low regime in cortex is emphasized by recent evidence that suggests that these slow oscillations play a role in synchronizing faster activity and modulating cortical excitability (Hiltunen et al., 2014; Leong et al., 2018). The commercial availability of DC coupled amplifiers allowed clinicians to explore bandwidths outside of the traditionally defined analog limit of $0.5-50 \mathrm{~Hz}$ in clinical neurofeedback. Training these very low frequencies was dubbed infraslow fluctuation (ISF) training due to the interaction of frequency and DC potential shift (Smith, Collura, Ferrara, \& de Vries, 2014).

The clinician's primary goal in ISF training is to find a client's optimum frequency (OF). Clinical experience has shown that this results in the best treatment response. Electrodes are placed at specific locations according to the internationally recognized 10/20 system (Silverman, 1963; Wei, Wu, \& Tudor, 2017). To begin the optimization process, one of three potential bipolar montage sites are chosen depending on the results of the quantitative electroencephalogram ( $q E E G$ ) and the client's chief complaint.

During ISF training the client experiences emergent state shifts that we refer to as symptoms of training. The symptoms that clients experience during and after the training session guide the clinician to the OF. Many clinicians use peripheral biofeedback measurements like skin temperature, skin conductance, or electromyography (EMG) as a guide to optimization as well. Once the optimum frequency is identified clients report improved sleep regulation, anxiety reduction, better appetite awareness and control, improved sexual function, and reduced reactivity to sound-all indications of improved sympathetic-parasympathetic response (Fink, Bronas, \& Calik, 2018; McCorry, 2007).

The ANS has a central role in maintaining sympathetic-parasympathetic and cardiovascular homeostasis. It includes vagal cholinergic and sympathetic noradrenergic nerves supplying the heart and sympathetic noradrenergic nerves that enmesh arterioles. These nerves play a role in peripheral resistance to blood flow in the body, and therefore blood pressure. That is why clinicians and researchers have long sought valid, noninvasive, quantitative means to identify pathophysiologically relevant abnormalities of these systems (Goldstein, Bentho, Park, \& Sharabi, 2011).

Heart rate variability (HRV) is one of the best-known means of physiological measurement and is defined as the beat to beat variability in the sinus rhythm over time. There is mounting evidence that points to the efficacy of HRV training in clinical practice due to its impact on sympathetic-parasympathetic function (Peper, Harvey, Lin, Tylova, \& Moss, 2015). The monitoring of sympathetic-parasympathetic response during ISF neurofeedback training may aid in the identification of the client's optimum frequency (Camp, Remus, Kalburgi, Porterfield, \& Johnson, 2012; Collura, 2013). Measurements of high frequency (HF) and low frequency (LF) power are good indicators of changes in HRV. It has been shown that HF mainly reflects respiratory sinus arrhythmia and HF HRV is understood to reflect the parasympathetic branch of the ANS (Akselrod et al., 1981; Stein, Bosner, Kleiger, \& Conger, 1994). LF is 
not as well understood, but it is believed that LF indicates baroreflex functionality and cardiac autonomic outflow (Goldstein et al., 2011). LF is often described in the literature as reflecting a combination of sympathetic and parasympathetic influences. HRV can be a valuable tool in the estimation of autonomic status especially when combined with other peripheral nervous system measures (Li, Rüdiger, \& Ziemssen, 2019).

Studies propose that specific emotions may elicit distinct autonomic functions. Biofeedback measures may correlate with specific emotions; for example, temperature, HR, and skin conductance may correlate to fear, joy, sadness, and anger. This suggests that the correlation of emotional state with autonomic response may be possible through the implementation of biofeedback methods (Collet, Vernet-Maury, Delhomme, \& Dittmar, 1997; Levenson, 1992).

Infraslow training is a recent addition to the relatively young field of neurofeedback. While the literature is growing rapidly (Leong et al., 2018; Mathew, Adhia, Smith, De Ridder, \& Mani, 2020; Smith, 2013; Smith et al., 2014; Smith, Leiderman, \& de Vries, 2017), the physiological systems involved and full impact on the body are not yet well understood. The aim of this research is threefold: (1) to contribute to the methods' scientific validity and eliminate speculation as to the physiological systems involved, (2) to identify the measurable responses that reveal the most sensitive and so the fastest path to optimization, and (3) to identify the best objective measure to help clients who lack subjective awareness of their physiological responses.

\section{Methods}

\section{Participants and Setting}

Sampling was randomized (Figure 1) and consisted of a population group that had already decided of their own volition to do neurofeedback training. These participants (Table 1) were randomly assigned to either the ISF or control group. All neurofeedback training was completed in a clinical setting.

Approval from the University of Pretoria Health Sciences Ethical Committee was obtained prior to the start of the study, and all participants completed an informed consent document.

\section{Inclusion/Exclusion Criteria}

\section{Inclusion criteria.}

- Reports of high anxiety.

- Reports of daily problems with concentration and memory.

- Complaints of sleep problems.

- Reports of emotional or anger control problems.

- Subjects that were able to participate in at least two training sessions per week.

- Subjects that completed a minimum of 10 sessions of neurofeedback training.

\section{Exclusion criteria.}

- Subjects using recreational drugs.

- Subjects using benzodiazepines.

- Subjects using any new form of medication during the study period that may influence the outcome.

- Subjects unable to complete a minimum of 10 training sessions.

- Subjects unable to attend two training sessions per week.

\section{Screening Questionnaires}

The subjects completed a clinical interview and an evaluation questionnaire to determine current function. The questionnaire was used by all ISF practitioners participating in the study to develop a broad range of information on their subjects.

From clinical interviews and the questionnaire, it was determined that the five main complaints shared by participants were:

- Anxiety/panic attacks (19 participants)

- Difficulty with either falling asleep or maintaining sleep (16 participants)

- Irritation/anger problems (7 participants)

- Executive function problems: concentration/ focus/memory complaints (20 participants)

- Constipation and or irritable bowls (6 participants) 


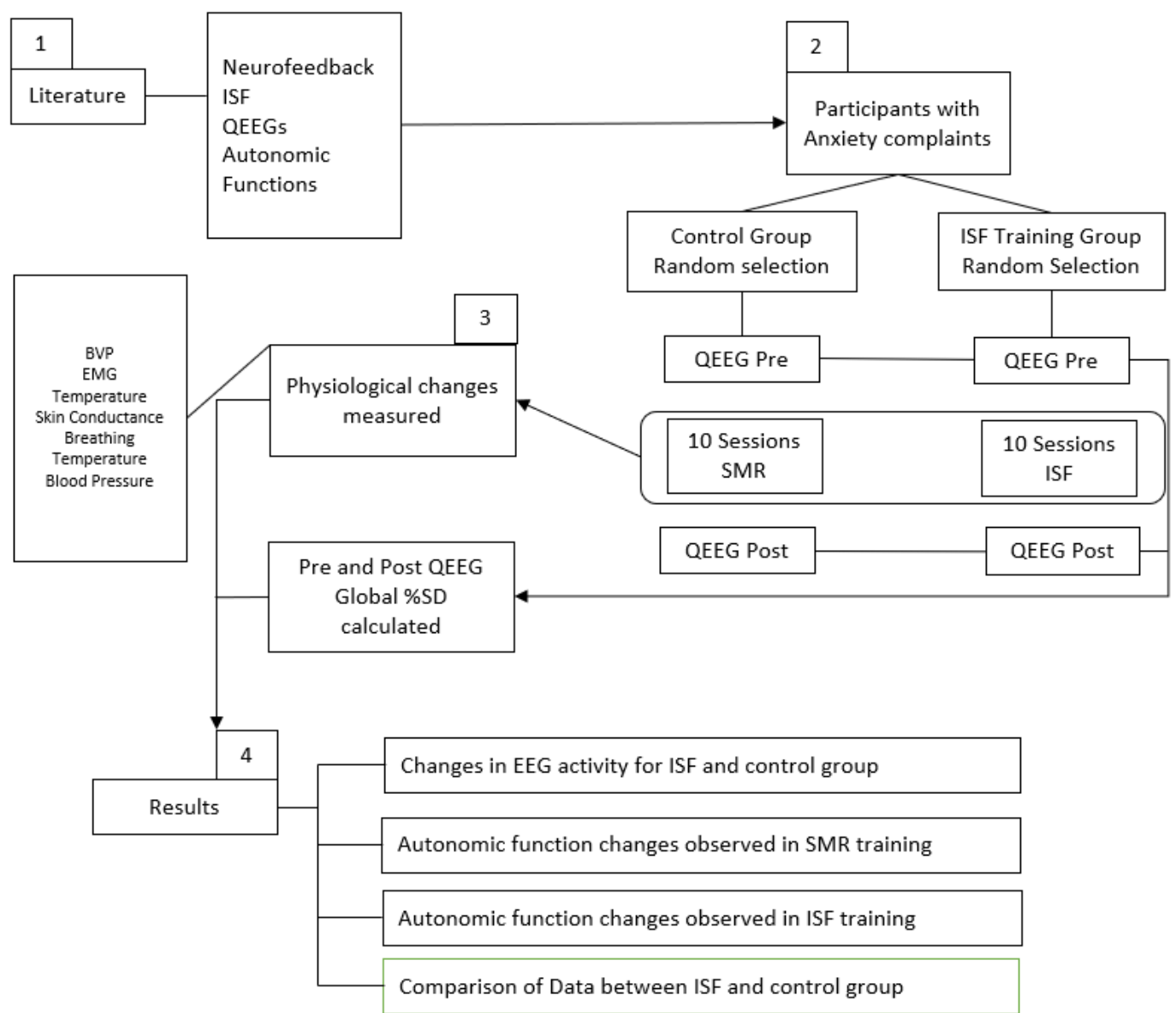

Figure 2. Consort flow chart.

\section{Table 1}

Participant demographics

\begin{tabular}{cccc}
\hline Subject & Sex & Handedness & Age (Years) \\
\hline C1 & M & Left & 36 \\
C2 & M & Right & 39 \\
C3 & M & Right & 26 \\
C4 & F & Right & 36 \\
C5 & M & Left & 19 \\
C6 & M & Right & 55 \\
C7 & M & Right & 18 \\
C8 & F & Right & 55 \\
C9 & M & Left & 26 \\
S1 & M & Right & 35 \\
S2 & M & Right & 22 \\
S3 & F & Right & 23 \\
S4 & F & Right & 24 \\
S5 & M & Right & 49 \\
S6 & F & Left & 36 \\
\hline
\end{tabular}

\begin{tabular}{cccc}
\hline Subject & Sex & Handedness & Age (Years) \\
\hline S7 & F & Right & 23 \\
S8 & F & Right & 23 \\
S9 & M & Right & 37 \\
S10 & M & Right & 24 \\
S11 & M & Right & 48 \\
S12 & M & Right & 49 \\
S13 & F & Right & 38 \\
S14 & M & Right & 22 \\
S15 & M & Right & 55 \\
S16 & M & Right & 38 \\
S17 & F & Right & 49 \\
S18 & F & Right & 34 \\
S19 & F & Right & 27 \\
S20 & F & Right & 18 \\
\hline Total Male & 17 & Mean Age & 33.9 \\
Total Female & 12 & - Control & 34.4 \\
& & - Experimental & 33.7 \\
\hline
\end{tabular}




\section{Physiological Data Monitoring}

The ProComp Infiniti (Thought Technology Ltd., Montreal, Canada) was developed for the intended purpose of biofeedback, relaxation, and muscle reeducation. It is an 8-channel multimodality device that provides real-time computerized biofeedback and data acquisition. The sampling rate is 2048 samples per second in two of the channels and 256 samples per second in the other six channels. The encoder can be used to render a wide range of physiological signs to be used in clinical observation as well as biofeedback.

The following measures were used in the study:

- Electromyography (EMG)

- Skin conductance

- Skin temperature

- Blood volume pulse (BVP), HR, and amplitude

- Breathing rate

- Blood pressure (Braun VitalScan 3 Wrist blood pressure monitor)

The BioGraph Infiniti software version 6.0 (Thought Technology Ltd., Montreal, Canada) allows simultaneous monitoring of the above mentioned physiological parameters (Huster, Mokom, EnriquezGeppert, \& Herrmann, 2014).

The EMG triode sensor was placed on the trapezius muscle on the right shoulder. The temperature sensor was fastened to the right thumb with a Velcro strap for all clients. The skin conductance sensor consists of two sensors that were placed on the second and third fingers. The BVP sensor was placed on the ring finger also with a Velcro strap. Breathing rate and amplitude was measured using a strap around the abdomen. The resting blood pressure was measured before and after each session while the participant remained in a seated position by making use of a wrist cuff and the values for systolic and diastolic blood pressure were recorded in millimeters of mercury $(\mathrm{mmHg}$; Huster et al., 2014).

No biofeedback training was done, and the client never saw the physiological measurements so that they were never cognitively aware of any changes taking place. The measurements were recorded for $34 \mathrm{~min}$, of which $30 \mathrm{~min}$ was the length of an ISF training session and 2 min pre- and postsession.

\section{ISF Neurofeedlback}

The Discovery 24-channel EEG amplifier (BrainMaster Technologies, Inc., Bedford, $\mathrm{OH}$ ) is a physiological monitoring and feedback system. It offers monitoring and feedback of brain signals that include the measurement of EEG, direct current, and slow cortical potentials (DC/SCP).

The BrainMaster Atlantis $4 \times 4$ (BrainMaster Technologies, Inc., Bedford, OH) 4-channel EEG amplifier is a physiological monitoring and feedback system. Just like the BrainMaster Discovery, it offers monitoring and feedback of EEG and DC/SCP. Each area on the scalp was cleaned using NuPrep skin preparation gel. Electrodes were then pressed onto the cleaned area using 10/20 conductive paste.

Training was done using a bipolar montage. A twochannel linked ear electrode array was implemented. This allowed for Z-score and amplitude monitoring at each site in a linked ear montage while bipolar training was simultaneously accomplished in a third virtual channel. Two active electrodes were placed at either T3-T4 or T4-P4. Two references were placed on the mastoid bone behind the ears. The ground was placed centrally at the $\mathrm{Cz}$ position according to the International 10/20 System. The duration of a session was $30 \mathrm{~min}$.

Training was done via visual and auditory feedback. The subjects heard two reward sounds-a low tone when the amplitude decreased and a high tone when it increased. It is an instant reward and continues for the duration of the condition. There was no refractory period between rewards which allows for the rapid transmission of information to the subject concerning changes in the amplitude and phase of the ISF signal (Smith et al., 2014).

The objective in infraslow neurofeedback training is to find a frequency that produces a state of focus and relaxation within a client. Changes in frequency are guided by the changes in state that the subject experiences. These changes in state or "symptoms of training" reflect shifts along the autonomic spectrum. For instance, a subject may experience her eyes watering, a parasympathetic response, or her pupils becoming dilated, a sympathetic response. The therapist makes changes in frequency that reduce the targeted symptom of training and so moves a client closer to autonomic quiescence.

Recorded data was stored to later be statistically analyzed to track changes that occurred in session and from session to session. 


\section{Sensorimotor Rhythm (SMR)/Control Group} Each area on the scalp was cleaned using NuPrep skin preparation gel. Electrodes were then pressed onto the cleaned area using 10/20 conductive paste. Three electrodes were used. One active placed at the $\mathrm{C} 4$ site, one reference placed on the left mastoid, and a ground placed on the right mastoid. The duration of the training session was $30 \mathrm{~min}$. The client watched a movie as did clients in the experimental group. In the background they could hear a tone which sounded when all parameters were met. To aid as visual feedback, the screen was dimmed when the client was not meeting the required thresholds and brightened when conditions were met. Neurofeedback for the control group consisted of a sensorimotor rhythm (SMR) protocol that enhanced $12-15 \mathrm{~Hz}$ activity and inhibited $3.5-7.0 \mathrm{~Hz}$ and $20-30$ $\mathrm{Hz}$ activity. For some clients, an adjustment was made to reward $12-16 \mathrm{~Hz}$ as they reported feeling more focused with this frequency band.

The same set of physiological measurements as described for the ISF training group were gathered for the SMR training sessions. Like the experimental group, the control group's physiological monitoring was also recorded for the 30-min duration of the session with an additional 2 min pre- and postsession.

\section{Statistical Analysis}

The following measurements were taken and statistically analyzed to determine which changes show the most statistically relevant results:

BVP amplitude, BVP inter-beat-interval (IBI), BVP HR from IBI, BVP HR (smoothed), BVP IBI peak frequency, BVP very low frequency (VLF) \% power, BVP LF \% power, BVP HF \% power, respiratory amplitude (abdominal), respiration rate, $\mathrm{HR}$ max - HR min, BVP amplitude mean, BVP HR mean (beats/min), BVP peak frequency mean $(\mathrm{Hz}), \mathrm{BVP} \mid \mathrm{BI}$ standard deviation of the RR interval (SDRR), BVP VLF \% power mean, BVP LF \% power mean, BVP HF \% power mean, BVP VLF total power mean, BVP LF total power mean, BVP HF total power mean, BVP LF/HF (means), EMG mean $(\mu \mathrm{V})$, skin conductance mean $(\mu S)$, temperature mean (deg), respiratory rate mean (breaths/min), BVP IBI peak amplitude, BVP IBI peak amplitude trigger, BVP IBI NN intervals, BVP IBI percentage of $N N$ intervals $(p N N)$, BVP IBI $p N N$ intervals (\%).

All electronic data was stored on a laptop of the investigator, and daily backups were made to a cloud storage account.
All physiological data gathered in the BioGraph Infiniti software was scanned for any artefact. Identified artefact was removed before raw data was analyzed statistically.

The mean, median, standard deviation, and interquartile range were used to describe the statistical findings. To determine if there were trends in the ISF parameters over the 10 sessions, a linear mixed model was used where random intercept and slope effects as well as the difference between pre-post sessions were evaluated. The test was evaluated at a level of $5 \%$ significance, and all analysis was done with STATA 14.

\section{Results}

HRV Very Low Frequency (VLF) and Low Frequency (LF) Power

By making use of a fast Fourier transform (FFT) allorhythmia, the heart rhythm can be decomposed into different frequencies. This can be graphically represented and distinguishes HF, LF, and VLF. The amplitude of these frequencies is usually very different for an individual in a high stress state than for one who is relaxed (Figure 3).

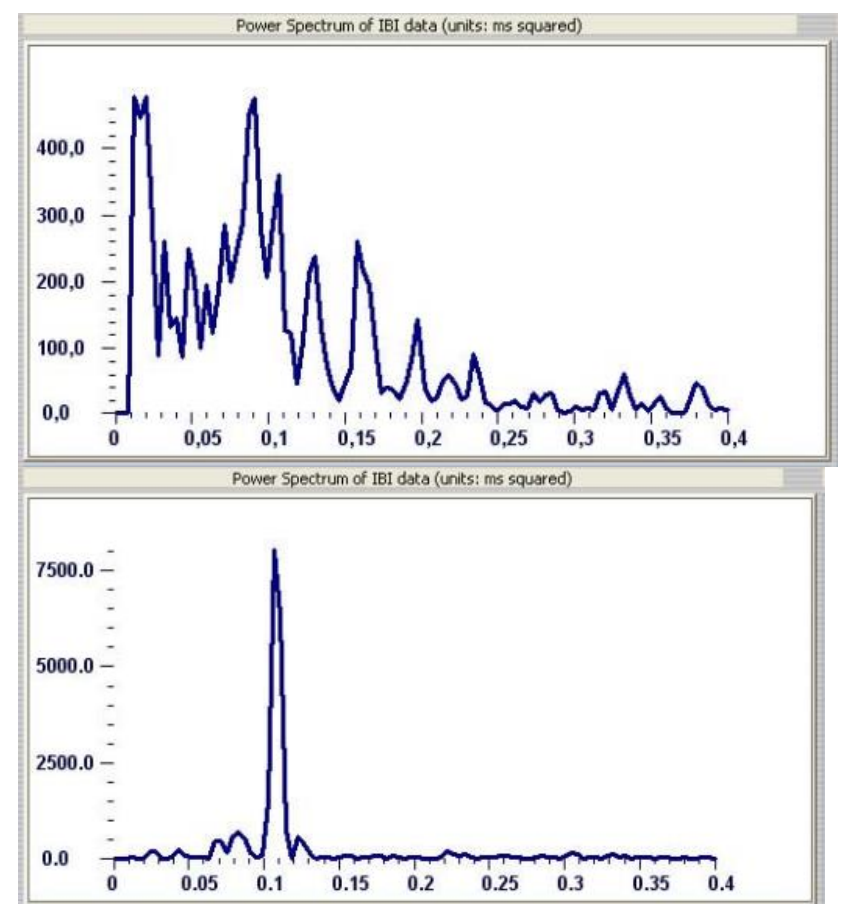

Figure 3. Comparison of HRV frequencies in a stress state (top) compared to a relaxed state (bottom; Thought Technology, 2014). 
HRV depends on the activity of the ANS. The VLF has been found to be a major determinant of physical activity, and it has also been proposed as a marker of sympathetic activity. It is said to affect the vascular tone loop of the baroreflex system, thermal regulation, and activity of the renin-angiotensin system. A decrease in the VLF is hypothesized to be an indication of sympathetic blocking (Sztajzel, 2004).

The LF is an indication of both sympathetic and parasympathetic activity and is indicative of activity of the baroreflex function. The HF is indicative of parasympathetic activity (Sztajzel, 2004). A decrease in $\mathrm{HF}$ is theorized to reflect decreased vagal activity leading to decreased parasympathetic activation (Rodin, Bornfleth, \& Johnson, 2017). There is controversy with respect to the ratio of LF to HF. Some consider it to be a measurement of balance between the sympathetic and parasympathetic nervous systems while others dispute that hypothesis (Eckberg, 1997; Malik, 1998).

When looking at the $t$-test results for the different components when comparing session 1 to session 10 the following values were obtained:

- VLF, $p=.05$. This indicates a significant decrease in the VLF \% power. This may be an indication of decreased sympathetic activity as it is hypothesized that increased VLF represents sympathetic blocking (van der Kruijs et al., 2016).

- LF, $p=.004$. This indicates a significant increase in the LF \% power. It reflects sympathetic and vagal influences on cardiac control via baroreceptor-mediated regulation of blood pressure according to some theories. Others suggest that it is a marker for sympathetic modulation (Prinsloo et al., 2011). The range for LF frequency is 0.04 $0.15 \mathrm{~Hz}$ with PNS dominance believed to be from $0.04-0.70 \mathrm{~Hz}$. When looking at the BVP peak mean frequency measured, the peak mean frequency measured at session 1 was $0.1 \mathrm{~Hz}$ and this decreased to $0.08 \mathrm{~Hz}$ at session 10. Due to it appearing insignificant the data was not further included in the larger results, but it does however indicate a possible shift towards being more parasympathetic dominant.

\section{EMG Mean}

Anxiety is often accompanied by muscle over-activity (Pluess, Conrad, \& Wilhelm, 2009). The amplitude of action potentials recorded from the muscles of individuals with anxiety is higher than healthy controls. One would expect a decrease in muscle tension with relaxation and parasympathetic activation (Barrett, Barman, Boitano, \& Brooks, 2012).

At rest there should be very low muscle activity as no muscle fibres are being recruited. Under chronic or repeated stress, certain muscles may not be able to return to a resting state. This is known as "residual" muscle tension and commonly causes back, shoulder, or neck pain. This can lead to increased fatigue (Sainsbury \& Gibson, 1954). A high resting muscle tension also leads to an impedance of blood flow which slows down healing. Adaptive habits such as muscle guarding can also have a negative impact by reducing flexibility and decreasing muscular efficiency (Sainsbury \& Gibson, 1954).

In the control group a change was seen in the positive direction when comparing session 1 to session 10 . However, it was not statistically significant.

The $p$-value for EMG in the experimental group is $p$ $=.01$ when comparing session 1 to session 10 , which shows a significant decrease in muscle tension and more specifically the resting muscle tension.

\section{Skin Conductance Mean}

Skin conductance is generally accepted as one of the most sensitive measures of emotional arousal (Lin, Lin, Lin, \& Huang, 2011). Skin conductance biofeedback is used to help an individual become aware of unconscious physiological responses to stress. A certain level of arousal is important, but if this increases too much it can lead to fatigue and anxiety as shown in Figure 4.

The $p$-value for skin conductance was $p<.0001$, which indicates a significant decrease in the experimental group when comparing session 1 to session 10. This is indicative of lower arousal levels, which is a positive change in a high anxiety population group where very high arousal levels are typically noted. There is also a decrease noted in the standard deviation of the group as well as a decrease of the median. This can be an indication of decreased anxiety as an increase in electrodermal activity is commonly reported in high anxiety individuals. 


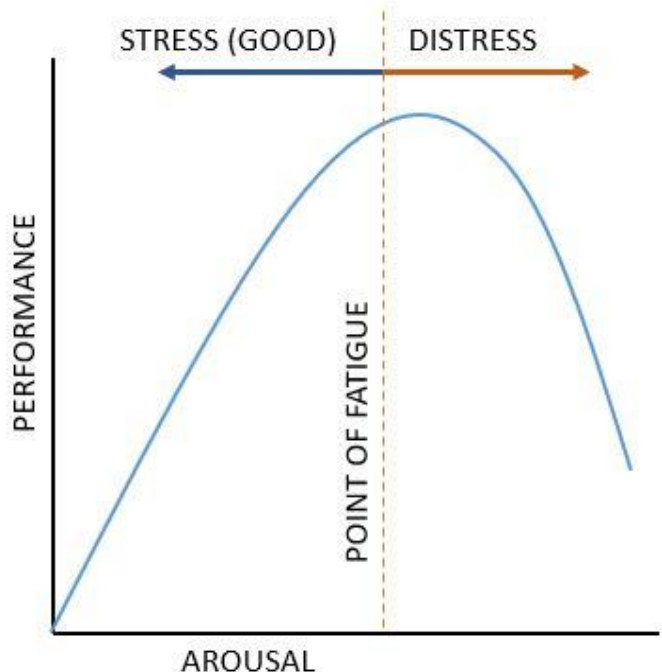

Figure 4. How stress and arousal influences performance.

\section{Blood Pressure}

The normal blood pressure in the brachial artery is approximately $120 / 70 \mathrm{mmHg}$ in young adults (Figure 5). It is affected by cardiac output, peripheral resistance, and emotion. Individuals with hypertension were able to lower blood pressure by engaging in anxiety treatment (Grossman et al., 2005).

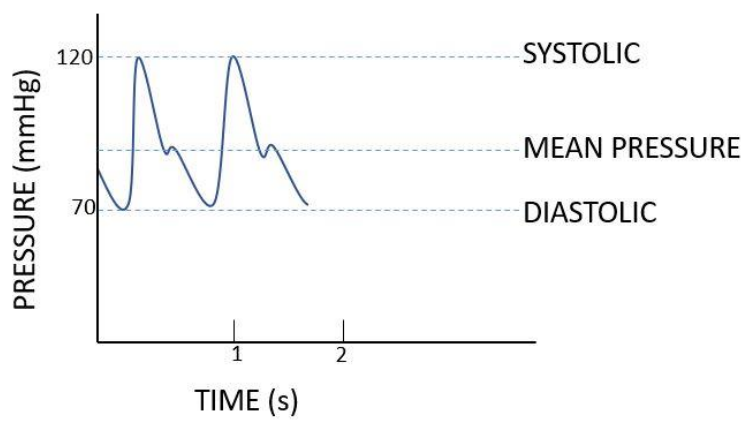

Figure 5. The Brachial artery pressure curve in a normal young adult indicating the relation of systolic and diastolic pressure to mean pressure.

There was a significant change noted in the post systolic comparison in the experimental group, $p$ value of .049. The diastolic value also approached a significant change, with a $p$-value of .083 . Brachial artery pressure monitoring reveals a significant effect on the blood pressure of participants when doing ISF training when compared to the control group where no significant changes were noted.
As with the heart rate analysis there was also a very large change in the standard deviation of the group indicating that we are dealing with a nonlinear change. There appears to be a homeostatic change towards what is considered normal (Figure 6).

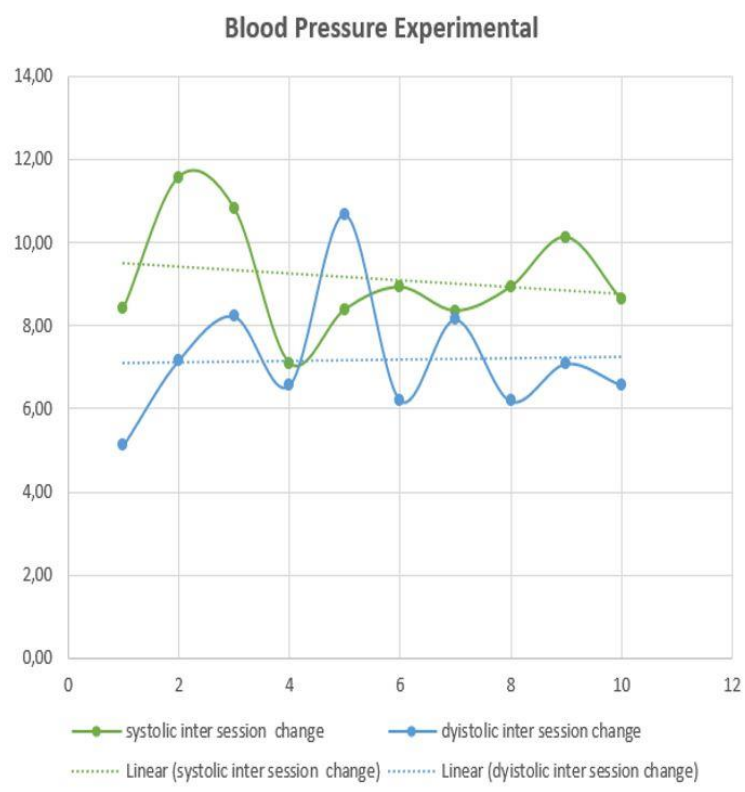

Figure 6a. In session change towards normal values for blood pressure experimental. $\mathrm{x}=$ session number, $\mathrm{y}=$ change towards normal.

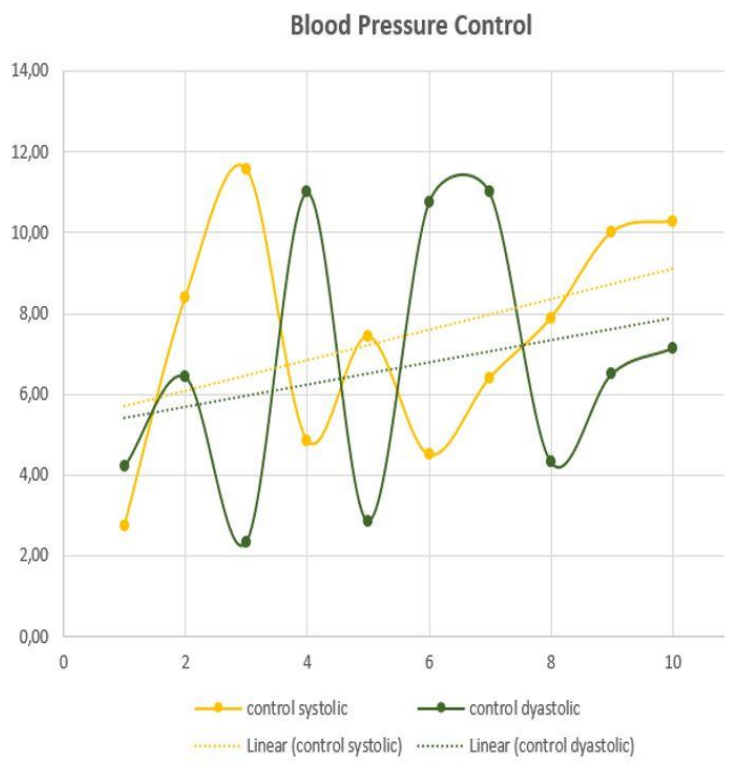

Figure 6b. In session change towards normal values for blood pressure control. $\mathrm{x}=$ session number, $\mathrm{y}=$ change towards normal. 


\section{Limitations of This Study and Recommendations} for Future Research

The size of the experimental and control groups defines this research as a pilot study. A larger study is warranted with a larger group of both experimental and control participants. There was no blinding involved in this study. A future study may include blinding of the rater and the subject. That will give an even clearer indication of what variables are purely influenced by the ISF training.

\section{Conclusion}

In the evaluation of the changes in autonomic function, significant changes were noted in the experimental group for blood pressure (systolic), skin conductance, electromyogram, and, in the analysis of the frequency domain of HRV, for \% power VLF and \% power LF. Table 2 summarizes the most significant $p$-values that were measured during the study. Heart rate data showing significant decrease was not noted, but when looking at the change towards normal a definite trend was seen wherein the standard deviation of the experimental group decreased and their heart rates either increased or decreased towards what is known as an age- and sex-related norm. The same was noted in the blood pressure readings, where the standard deviation of the experimental group decreased, and more participants BP values increased or decreased towards more normal values. In other words, closer to the normal range of $120 / 70$. No significant changes were observed in the control group.

\section{Table 2}

Summary of $p$-values obtained

\begin{tabular}{ccc}
\hline Variable & $\begin{array}{c}p \text {-value } \\
\text { ISF }\end{array}$ & $\begin{array}{c}p \text {-value } \\
\text { Amplitude (SMR) }\end{array}$ \\
\hline HRV VLF Power & .050 & .30 \\
HRV LF Power & .004 & .98 \\
EMG Mean & .010 & .33 \\
$\begin{array}{c}\text { Skin Conductance } \\
\text { Mean }\end{array}$ & $<.0001$ & .39 \\
$\begin{array}{c}\text { Blood Pressure } \\
\text { (Systolic) }\end{array}$ & .049 & .74 \\
$\begin{array}{c}\text { Blood Pressure } \\
\text { (Diastolic) }\end{array}$ & .083 & .79 \\
\hline
\end{tabular}

Physiological monitoring may be a useful tool in future studies to determine the ANS impact of neurofeedback training. In the present study, our data suggest that the mechanism of action of ISF neurofeedback training may involve the ANS. Our data also suggest that SMR training does not involve ANS function. While not unique in comparing one form of neurofeedback with another, the current study may be the first to provide data that differentiates the functional impact of one form of neurofeedback training from another. Finally, the results of this study suggest that peripheral biofeedback measures of electromyogram, skin conductance, the frequency bands of HRV, and blood pressure may help in the frequency optimization process of infraslow neurofeedback training. These peripheral measures help to clarify the physiological impact on clients who may lack the subjective awareness to report accurately.

\section{Author Declaration}

Mark Smith is the owner of Neurofeedback Therapy Services of New York. NTSNY produces neurofeedback workshops that include teaching infraslow fluctuation neurofeedback training. He provided neurofeedback protocols for this research and participated in editing of the final version of the manuscript. He did not participate in the research design, implementation, or evaluation of the data. All other authors declare no competing interests.

\section{References}

Akselrod, S., Gordon, D., Ubel, F. A., Shannon, D. C., Berger, A. C., \& Cohen, R. J. (1981). Power spectrum analysis of heart rate fluctuation: $A$ quantitative probe of beat-to-beat cardiovascular control. Science, 213(4504), 220-222. https://doi.org/10.1126/science.6166045

Aladjalova, N. A. (1957). Infra-slow rhythmic oscillations of the steady potential of the cerebral cortex. Nature, 179(4567), 957-959. http://dx.doi.org/10.1038/179957a0

Alshelh, Z., Di Pietro, F., Youssef, A. M., Reeves, J. M., Macey, P. M., Vickers, E. R., ... Henderson, L. A. (2016). Chronic neuropathic pain: It's about the rhythm. The Journal of Neuroscience, 36(3), 1008-1018. https://doi.org/10.1523 /jneurosci.2768-15.2016

Barrett, K. E., Barman, S. M., Boitano, S., \& Brooks, H. L. (2012). Ganong's review of medical physiology. New York, NY: McGraw Hill. https://www.amazon.com/Ganongs-ReviewMedical-Physiology-Science/dp/0071780033

Beissner, F., Meissner, K., Bär, K.-J., \& Napadow, V. (2013). The autonomic brain: An activation likelihood estimation metaanalysis for central processing of autonomic function. The Journal of Neuroscience, 33(25), 10503-10511. https://doi.org/10.1523/jneurosci.1103-13.2013

Broyd, S. J., Helps, S. K., \& Sonuga-Barke, E. J. S. (2011). Attention-induced deactivations in very low frequency EEG oscillations: Differential localisation according to ADHD symptom status. PLOS ONE, 6(3), e17325. http://dx.doi.org /10.1371/journal.pone.0017325

Camp, R. M., Remus, J. L., Kalburgi, S. N., Porterfield, V. M., \& Johnson, J. D. (2012). Fear conditioning can contribute to 
behavioral changes observed in a repeated stress model. Behavioural Brain Research, 233(2), 536-544. https://doi.org /10.1016/j.bbr.2012.05.040

Collet, C., Vernet-Maury, E., Delhomme, G., \& Dittmar, A. (1997). Autonomic nervous system response patterns specificity to basic emotions. Journal of the Autonomic Nervous System, $62(1-2), \quad 45-57 . \quad$ https://doi.org/10.1016/S01651838(96)00108-7

Collura, T. F. (2013). Technical foundations of neurofeedback. New York, NY: Routledge/Taylor \& Francis Group.

Eckberg, D. L. (1997). Sympathovagal balance. Circulation, 96(9), 3224-3232. https://doi.org/10.1161/01.CIR.96.9.3224

Fink, A. M., Bronas, U. G., \& Calik, M. W. (2018). Autonomic regulation during sleep and wakefulness: A review with implications for defining the pathophysiology of neurological disorders. Clinical Autonomic Research, 28(6), 509-518. https://doi.org/10.1007/s10286-018-0560-9

Goldstein, D. S., Bentho, O., Park, M.-Y., \& Sharabi, Y. (2011). Low-frequency power of heart rate variability is not a measure of cardiac sympathetic tone but may be a measure of modulation of cardiac autonomic outflows by baroreflexes. Experimental Physiology, 96(12), 1255-1261. https://doi.org /10.1113/expphysiol.2010.056259

Grossman, E., Nadler, M., Sharabi, Y., Thaler, M., Shachar, A., \& Shamiss, A. (2005). Antianxiety treatment in patients with excessive hypertension. American Journal of Hypertension, 18(9), 1174-1177. https://doi.org/10.1016 /j.amjhyper.2005.03.728

Helfrich, R. F., Mander, B. A., Jagust, W. J., Knight, R. T., \& Walker, M. P. (2018). Old brains come uncoupled in sleep: Slow wavespindle synchrony, brain atrophy, and forgetting. Neuron, 97(1), 221-230.e4. https://doi.org/10.1016 /j.neuron.2017.11.020

Hiltunen, T., Kantola, J., Abou Elseoud, A., Lepola, P., Suominen, K., Starck, T., ... Palva, J. M. (2014). Infra-slow EEG fluctuations are correlated with resting-state network dynamics in fMRI. The Journal of Neuroscience, 34(2), 356-362. https://doi.org/10.1523/jneurosci.0276-13.2014

Huster, R. J., Mokom, Z. N., Enriquez-Geppert, S., \& Herrmann, C. S. (2014). Brain-computer interfaces for EEG neurofeedback: Peculiarities and solutions. International Journal of Psychophysiology, 91(1), 36-45. https://doi.org /10.1016/j.ijpsycho.2013.08.011

Joshi, R. B., Duckrow, R. B., Goncharova, I. I., Gerrard, J. L., Spencer, D. D., Hirsch, L. J., ... Zaveri, H. P. (2018). Seizure susceptibility and infraslow modulatory activity in the intracranial electroencephalogram. Epilepsia, 59(11), 20752085. https://doi.org/10.1111/epi.14559

Lecci, S., Fernandez, L. M. J., Weber, F. D., Cardis, R., Chatton, J.-Y., Born, J., \& Lüthi, A. (2017). Coordinated infraslow neural and cardiac oscillations mark fragility and offline periods in mammalian sleep. Science Advances, 3(2), e1602026. https://doi.org/10.1126/sciadv.1602026

Leong, S. L., Vanneste, S., Lim, J., Smith, M., Manning, P., \& De Ridder, D. (2018). A randomised, double-blind, placebocontrolled parallel trial of closed-loop infraslow brain training in food addiction. Scientific Reports, 8(1), 11659. https://doi.org/10.1038/s41598-018-30181-7

Levenson, R. W. (1992). Autonomic nervous system differences among emotions. Psychological Science, 3(1), 23-27. https://doi.org/10.1111/j.1467-9280.1992.tb00251.x

Li, K., Rüdiger, H., \& Ziemssen, T. (2019). Spectral analysis of heart rate variability: Time window matters. Frontiers in Neurology, 10(545). https://doi.org/10.3389/fneur.2019.00545

Lin, H.-P., Lin, H.-Y., Lin, W.-L., \& Huang, A. C.-W. (2011). Effects of stress, depression, and their interaction on heart rate, skin conductance, finger temperature, and respiratory rate: Sympathetic-parasympathetic hypothesis of stress and depression. Journal of Clinical Psychology, 67(10), 10801091. https://doi.org/10.1002/jclp.20833
Malik, M. (1998). Sympathovagal balance: A critical appraisal. Circulation, 98(23), 2643-2644. https://doi.org/10.1161 101.CIR.98.23.2643

Marshall, L., Mölle, M., Fehm, H. L., \& Born, J. (2008). Changes in direct current (DC) potentials and infra-slow EEG oscillations at the onset of the luteinizing hormone (LH) pulse. European Journal of Neuroscience, 12(11), 3935-3943. https://doi.org/10.1046/j.1460-9568.2000.00304.x

Mathew, J., Adhia, D. B., Smith, M. L., De Ridder, D., \& Mani, R. (2020). Protocol for a pilot randomized sham-controlled clinical trial evaluating the feasibility, safety, and acceptability of infraslow electroencephalography neurofeedback training on experimental and clinical pain outcomes in people with chronic painful knee osteoarthritis. NeuroRegulation, 7(1), 30-44. https://doi.org/10.15540/nr.7.1.30

McCorry, L. K. (2007). Physiology of the autonomic nervous system. American Journal of Pharmaceutical Education, 71(4), 78. https://doi.org/10.5688/aj710478

Palva, J. M., \& Palva, S. (2012). Infra-slow fluctuations in electrophysiological recordings, blood-oxygenation-leveldependent signals, and psychophysical time series. Neurolmage, 62(4), 2201-2211. https://doi.org/10.1016 /j.neuroimage.2012.02.060

Peper, E., Harvey, R., Lin, I.-M., Tylova, H., \& Moss, D. W. (2015). Is there more to blood volume pulse than heart rate variability, respiratory sinus arrhythmia, and cardiorespiratory synchrony? Biofeedback, 35(2), 54-61. https://api.semanticscholar.org/CorpusID:15486681

Pluess, M., Conrad, A., \& Wilhelm, F. H. (2009). Muscle tension in generalized anxiety disorder: A critical review of the literature. Journal of Anxiety Disorders, 23(1), 1-11. https://doi.org /10.1016/j.janxdis.2008.03.016

Prinsloo, G. E., Rauch, H. G. L., Lambert, M. I., Muench, F., Noakes, T. D., \& Derman, W. E. (2011). The effect of short duration heart rate variability (HRV) biofeedback on cognitive performance during laboratory induced cognitive stress. Applied Cognitive Psychology, 25(5), 792-801. https://doi.org/10.1002/acp.1750

Rodin, E., Bornfleth, H., \& Johnson, M. (2017). DC-EEG recordings of mindfulness. Clinical Neurophysiology, 128(4), 512-519. https://doi.org/10.1016/j.clinph.2016.12.031

Sainsbury, P., \& Gibson, J. G. (1954). Symptoms of anxiety and tension and the accompanying physiological changes in the muscular system. Journal of Neurology, Neurosurgery, \& Psychiatry, 17(3), 216-224. https://doi.org/10.1136 /jnnp.17.3.216

Silverman, D. (1963). The rationale and history of the 10-20 system of the International Federation. American Journal of EEG Technology, 3(1), 17-22. https://doi.org/10.1080 /00029238.1963.11080602

Smith, M. L. (2013). Infra-slow fluctuation training; On the downlow in neuromodulation. NeuroConnections, Fall, 38 \& 42.

Smith, M. L., Collura, T. F., Ferrara, J., \& de Vries, J. (2014). Infraslow fluctuation training in clinical practice: A technical history. NeuroRegulation, 1(2), 187-207. https://doi.org /10.15540/nr.1.2.187

Smith, M. L., Leiderman, L., \& de Vries, J. (2017). Infra-slow fluctuation (ISF) for autism spectrum disorders. In T. F. Collura \& J. A. Frederick (Eds.), Handbook of clinical QEEG and neurotherapy. New York, NY: Routledge/Taylor \& Francis Group. https://www.taylorfrancis.com/books/e /9781315754093/chapters/10.4324\%2F9781315754093-42

Stein, P. K., Bosner, M. S., Kleiger, R. E., \& Conger, B. M. (1994). Heart rate variability: A measure of cardiac autonomic tone. American Heart Journal, 127(5), 1376-1381. https://doi.org /10.1016/0002-8703(94)90059-0

Sztajzel, J. (2004). Heart rate variability: A noninvasive electrodardiographic method to measure the autonomic nervous system. Swiss Medical Weekly, 135(35-36), 514-522. https://www.ncbi.nlm.nih.gov/pubmed/15517504 
Thayer, J. F., \& Lane, R. D. (2000). A model of neurovisceral integration in emotion regulation and dysregulation. Journal of Affective Disorders, 61(3), 201-216. https://doi.org/10.1016 /S0165-0327(00)00338-4

Thought Technology. (2014). BioGraph 6.0 Software User Manual. Montreal, Canada: Thought Technology Ltd.

van der Kruijs, S. J. M., Vonck, K. E. J., Langereis, G. R., Feijs, L. M. G., Bodde, N. M. G., Lazeron, R. H. C., ... Cluitmans, P. J. M. (2016). Autonomic nervous system functioning associated with psychogenic nonepileptic seizures: Analysis of heart rate variability. Epilepsy \& Behavior, 54, 14-19. https://doi.org/10.1016/j.yebeh.2015.10.014

Warren, S. M., Chou, Y.-H., \& Steklis, H. D. (2020). Potential for resting-state $\mathrm{fMRI}$ of the amygdala in elucidating neurological mechanisms of adaptive self-regulatory strategies: A systematic review. Brain Connectivity, 10(1), 3-17. https://doi.org /10.1089/brain.2019.0700

Wei, Y., Wu, Y., \& Tudor, J. (2017). A real-time wearable emotion detection headband based on EEG measurement. Sensors and Actuators A: Physical, 263, 614-621. https://doi.org /10.1016/j.sna.2017.07.012

Received: May 1, 2020

Accepted: May 12, 2020

Published: June 27, 2020 